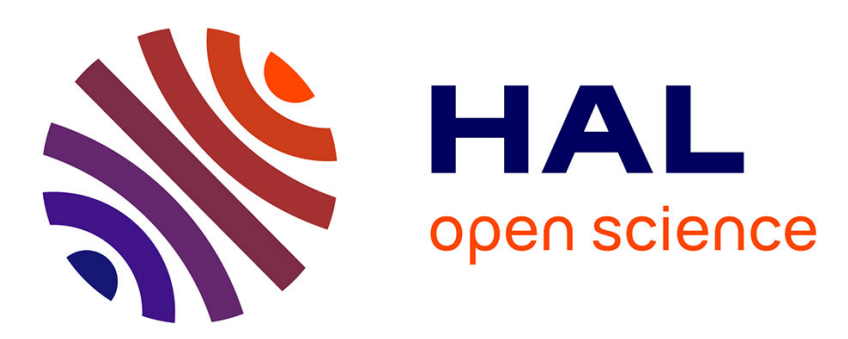

\title{
Topology Optimization in Electromagnetic Casting via quadratic programming
}

Alfredo Canelas, Jean Rodolphe Roche

\section{To cite this version:}

Alfredo Canelas, Jean Rodolphe Roche. Topology Optimization in Electromagnetic Casting via quadratic programming. 2012. hal-00758806

\section{HAL Id: hal-00758806 \\ https://hal.science/hal-00758806}

Preprint submitted on 29 Nov 2012

HAL is a multi-disciplinary open access archive for the deposit and dissemination of scientific research documents, whether they are published or not. The documents may come from teaching and research institutions in France or abroad, or from public or private research centers.
L'archive ouverte pluridisciplinaire HAL, est destinée au dépôt et à la diffusion de documents scientifiques de niveau recherche, publiés ou non, émanant des établissements d'enseignement et de recherche français ou étrangers, des laboratoires publics ou privés. 


\title{
Topology Optimization in Electromagnetic Casting via quadratic programming
}

\author{
Alfredo Canelas ${ }^{\mathrm{a} *}$ and Jean R. Roche ${ }^{\mathrm{b}}$ \\ ${ }^{a}$ Instituto de Estructuras y Transporte, Facultad de Ingeniería, UDELAR, J. Herrera y \\ Reissig 565, CP 11300, Montevideo, Uruguay. \\ ${ }^{\mathrm{b}}$ Institut Elie Cartan de Nancy, Université de Lorraine, CNRS, INRIA, B.P. 70239, \\ 54506, Vandoeuvre lès Nancy, France.
}

$(* * * *)$

\begin{abstract}
A new optimization method is proposed for solving an inverse problem concerning the shape and topology of the inductors used in the electromagnetic casting technique of the metallurgical industry. The method is based on an sparse convex quadratic programming version of a recently proposed topology optimization formulation of the inverse electromagnetic casting problem. Regular 0-1 solutions are found by adding to the original Kohn-Vogelius objective function an appropriate penalty term that preserves the quadratic programming structure of the problem, allowing the use of efficient interior-point algorithms.

Results for some numerical examples are presented, showing that the technique proposed is effective and can successfully find inductors of optimal shape and topology.
\end{abstract}

Keywords: inverse problem, topology optimization, electromagnetic casting, quadratic programming, interior-point methods

AMS Subject Classification: 49Q10; 90C90; 90C51; 65K10; 65R32

\section{Introduction}

This paper concerns the numerical solution of an inverse problem regarding electromagnetic casting (EMC) of molten metals. The EMC is an industrial technique that allows for contactless heating, shaping and controlling of chemical aggressive hot melts. It makes use of the repulsive forces that an electromagnetic field produces on the surface of a mass of liquid metal. Under suitable assumptions, the equilibrium configurations are described by a set equations expressing an equilibrium relation on the liquid metal boundary between the electromagnetic pressure and the surface tension forces. The equilibrium relation involves the curvature of the boundary and the solution of an elliptic exterior boundary value problem. The equilibrium shape is known to be a stationary state of the total energy functional under the constraint that the surface area is prescribed $[1,2]$.

The inverse EMC problem considered in this paper consists of determining the electric currents that induce the exterior magnetic field for which the liquid metal takes on a given desired shape. In previous works we studied the inverse EMC problem in the case that the inductors are single solid-core wires of negligible crosssection area, and the more realistic case where they are made of a set of bounded insulated strands $[3,4]$. In both cases the number of inductors was fixed in advance.

*Corresponding author. Email: acanelas@fing.edu.uy 
In a later article we overcame this limitation and looked for optimal configurations of inductors considering a topology optimization formulation based on the KohnVogelius criterion, which was solved by means of a topology optimization technique based on the topological derivative concept [5].

In the present paper we consider the topology optimization formulation proposed in [5] and state a simultaneous design and optimization (SAND) formulation of the inverse EMC problem. In this formulation we add a penalty term to the KohnVogelius criterion with the purpose of obtaining regular $0-1$ solutions. We show that the discrete version of the penalized SAND formulation is a convex quadratic programming problem that can be efficiently solved by using interior-point optimization algorithms. In addition to the interior-point algorithm, a simple variable mesh approach is proposed to further reduce the computational costs of solution.

The remaining contents of this paper are organized as following. Section 2 briefly describes the mathematical model of the EMC problem. Section 3 introduces the inverse EMC problem and describes the penalized SAND topology optimization formulation proposed and the variable mesh approach. Some numerical examples of two-dimensional exterior and interior EMC problems are studied in Section 4. Finally, the concluding remarks are given in Section 5.

\section{The mathematical model of the EMC problem}

The simplified model of the EMC problem studied here concerns the case of a vertical column of liquid metal falling down into an electromagnetic field created by vertical inductors. We consider the quasi-static model and assume that the frequency of the imposed electric current is very high, so that the magnetic field does not penetrate into the metal. In other words, we rely on the skin effect. Moreover, we assume that a stationary horizontal section is reached so that the two-dimensional model is valid. The equilibrium of the system is ensured by the static balance on the surface of the metal between the surface tension and the electromagnetic forces. This problem and other similar ones have been considered by several authors, we refer the reader to papers [1, 2, 6-11] for the physical analysis of the simplifying assumptions of the model.

Let $\Omega \subset \mathbb{R}^{2}$ be the exterior of the domain $\omega$ occupied by the cross-section of the metal column, which is assumed closed, simply connected, and with a non-void interior. Under these assumptions, the equilibrium surface $\Gamma$ of the molten metal column is characterized by the following equations [10, 12-15]:

$$
\begin{aligned}
\nabla \times \boldsymbol{B} & =\mu_{0} \boldsymbol{J} & & \text { in } \Omega, \\
\nabla \cdot \boldsymbol{B} & =0 & & \text { in } \Omega, \\
\boldsymbol{B} \cdot \nu & =0 & & \text { on } \Gamma, \\
\|\boldsymbol{B}(x)\| & =O\left(\|x\|^{-1}\right) & & \text { as }\|x\| \rightarrow \infty \text { in } \Omega, \\
\frac{1}{2 \mu_{0}}\|\boldsymbol{B}\|^{2}+\sigma \mathcal{C} & =p_{0} & & \text { on } \Gamma .
\end{aligned}
$$

Here the fields $\boldsymbol{J}=\left(0,0, j_{0}\right)$ and $\boldsymbol{B}=\left(B_{1}, B_{2}, 0\right)$ represent the mean square values of the electric current density vector and the total magnetic field, respectively. The constant $\mu_{0}$ is the vacuum permeability, $\nu$ is the unit normal vector to the boundary $\Gamma$ and $\|\cdot\|$ denotes the Euclidean norm. In (5) $\mathcal{C}$ is the curvature of $\Gamma$ seen from the metal, $\sigma$ is the surface tension of the liquid and the constant $p_{0}$ is an unknown of the problem. Physically, $p_{0}$ represents the difference between the 
internal and external pressures. Equations (1)-(5) correspond to the exterior EMC problem. In the interior EMC problem $\Omega$ is considered open, bounded an simply connected, and (4) is discarded. In both cases we assume that $j_{0}$ has a compact support in $\Omega$ and that the total electric current is zero:

$$
\int_{\Omega} j_{0} d x=0
$$

In the exterior EMC problem we assume that the cross-section area of the liquid metal column is known and equal to $S_{0}$ :

$$
\int_{\omega} d x=S_{0}
$$

while, in the case of the interior problem, $\Omega$ replaces $\omega$ in $(7)$.

Equations (1)-(6), with the function $j_{0}$ compactly supported in $\Omega$, imply the existence of the flux function $\varphi: \Omega \rightarrow \mathbb{R}$ such that $\boldsymbol{B}=\left(\frac{\partial \varphi}{\partial x_{2}},-\frac{\partial \varphi}{\partial x_{1}}, 0\right)$. Then, the boundary value problem regarding the inverse EMC problem in terms of the flux function $\varphi: \Omega \rightarrow \mathbb{R}$ is:

$$
\left\{\begin{aligned}
-\Delta \varphi & =\mu_{0} j_{0} & & \text { in } \Omega \\
\varphi & =0 & & \text { on } \Gamma, \\
\varphi(x) & =c+o(1) & & \text { as }\|x\| \rightarrow \infty
\end{aligned}\right.
$$

where the constant $c$ is the value at infinity of the solution $\varphi$ in the case of the exterior problem, which is also an unknown of (8) [3-5], while the condition at infinity is not considered in the interior EMC problem. Equivalent formulations of the condition at infinity are $\varphi(x)=O(1)$ and $\varphi(x)=c+O\left(\|x\|^{-1}\right)[16]$. The form used in (8) is convenient in the development of numerical methods of solution.

Problem (8) has unique solutions $\varphi \in W_{0}^{1}(\Omega)$ and $c \in \mathbb{R}[16,17]$, where $W_{0}^{1}(\Omega)$ is defined as:

$$
W_{0}^{1}(\Omega)=\left\{u: \beta u \in L^{2}(\Omega) \text { and } \nabla u \in L^{2}(\Omega)\right\},
$$

with $\beta(x)=\left[\sqrt{1+\|x\|^{2}} \log \left(2+\|x\|^{2}\right)\right]^{-1}$ in the exterior EMC problem and $\beta=1$ in the interior one. The equilibrium of the liquid metal boundary in terms of the flux function is:

$$
\frac{1}{2 \mu_{0}}\left|\frac{\partial \varphi}{\partial n}\right|^{2}+\sigma \mathcal{C}=p_{0} \quad \text { on } \Gamma .
$$

In the direct problem the electric current density $j_{0}$ and the cross-section area $S_{0}$ are given, and one has to find the shape of $\omega$ satisfying (7) such that the flux function $\varphi$ solution to (8) satisfies also the equilibrium equation (10) for a real constant $p_{0}$.

\section{The inverse EMC problem}

For clarity of exposition, we restrict the description of the inverse problem formulation to the case of the exterior EMC problem. The formulation of the interior problem is deduced following the same steps. The goal of the inverse problem is to find a distribution of electric current around the liquid metal column so that 
it attains a given shape. Therefore, we have to determine the electric current density $j_{0}$ satisfying (6) such that the solution $\varphi$ of (8) satisfies also the equilibrium equation (10). There are a few number of papers about the existence of exact solutions to the inverse problem, see $[7,18]$. Although these above-mentioned references constitute an important insight on the existence issue, we are also interested in obtaining approximate solutions in situations where the existence of solutions cannot be ensured. If the inverse problem has an exact solution, we say that the target shape is shapable, if it does not, we say that it is not shapable. Even considering a shapable shape, the inverse problem is inherently ill-posed: small variations of the liquid boundary may cause dramatic variations in the solution $j_{0}$ of the inverse problem $[7,18]$. In addition, the uniqueness of the solution in terms of $j_{0}$ cannot be ensured [5]. Hence, we follow the approach proposed in [5], where the inverse problem is formulated as an optimization problem, in order to look for a solution (maybe just an approximate solution) minimizing an appropriate functional.

There are, however, some known facts about the exact solutions of the inverse problem that are of main importance in what follows. From (5) and (10) we realize that $p_{0}$ must satisfy

$$
p_{0} \geq \max _{\Gamma} \sigma \mathcal{C}
$$

to have the field $\|\boldsymbol{B}\|$ well determined on $\Gamma$ by the boundary conditions (3) and $(5)[5,7]$. In addition, to satisfy $(3) \Gamma$ must be an analytic curve. If we want to find $j_{0}$ satisfying (6), it is necessary that $p_{0}=\max _{\Gamma} \sigma \mathcal{C}$. In that case the curvature $\mathcal{C}$ of a shapable domain $\omega$ must reach its maximum value at an even number of points [7].

Given $p_{0}$ by the equality in (11), the equilibrium equation reads

$$
\frac{\partial \varphi}{\partial n}=\varkappa \bar{p} \quad \text { on } \Gamma
$$

where $\bar{p}=\sqrt{2 \mu_{0}\left(p_{0}-\sigma \mathcal{C}\right)}$, and $\varkappa= \pm 1$, with the sign changes located at points where the curvature of $\Gamma$ is a global maximum. The two possible ways of defining $\varkappa$ lead to the same solution $j_{0}$ but with the opposite sign [5]. Therefore, we assume from now on that $\varkappa \bar{p}$ is a known function defined on $\Gamma$.

It is also possible to introduce some constraints in the position of the inductors to have realizable solutions. Considering a shapable $\omega$, Henrot and Pierre [7] proved that a solution with the electric current concentrated on a closed curve located at a small enough distance from $\Gamma$ can always be found.

\subsection{Problem Formulation}

The considerations made in Section 3 allow us to formulate the inverse problem as follows: for a given domain $\Omega$ limited by a smooth boundary $\Gamma$, determine an electric current density $j_{0}$ satisfying (6) with compact support $\operatorname{spt}\left(j_{0}\right) \subset \Theta$, where $\Theta$ is a given compact in $\Omega$ [7], and a real constant $c$ such that the system

$$
\left\{\begin{aligned}
-\Delta \varphi & =\mu_{0} j_{0} & & \text { in } \Omega, \\
\varphi & =0 & & \text { on } \Gamma, \\
\frac{\partial \varphi}{\partial n} & =\varkappa \bar{p} & & \text { on } \Gamma, \\
\varphi(x) & =c+o(1) & & \text { as }\|x\| \rightarrow \infty,
\end{aligned}\right.
$$


has a solution $\varphi \in W_{0}^{1}(\Omega)$. Therefore, a necessary condition for the existence of a solution to the inverse problem is the following [5]:

$$
\int_{\Gamma} \varkappa \bar{p} d s=0
$$

Let us introduce a shape functional based on the Kohn-Vogelius criterion, namely

$$
J(\phi)=\frac{1}{2}\|\phi\|_{L^{2}(\Gamma)}^{2}=\frac{1}{2} \int_{\Gamma} \phi^{2} d s
$$

where the auxiliary function $\phi$ depends implicitly on $j_{0}$ and $c$ by solving the following boundary-value problem

$$
\left\{\begin{aligned}
-\Delta \phi & =\mu_{0} j_{0} & & \text { in } \Omega, \\
\frac{\partial \phi}{\partial n} & =\varkappa \bar{p} & & \text { on } \Gamma, \\
\phi(x) & =c+o(1) & & \text { as }\|x\| \rightarrow \infty .
\end{aligned}\right.
$$

Note that (16) has a unique solution in $W_{0}^{1}(\Omega)$ if and only if the compatibility condition (14) is satisfied [16, 19].

The approach proposed in [5] to deal with (13) is the following: determine the electric current density $j_{0}$ and the constant $c$ such that the solution $\phi$ of (16) minimizes the shape functional (15). We note that the minimum of the shape functional (15) is attained when $\phi \equiv 0$ on $\Gamma$. This means that in this situation, from the well-posedness of problems (8) and (16), we have $\phi \equiv \varphi$ in $\Omega$.

A SAND approach for the previous problem can be obtained considering $j_{0}$ and $\phi$ as independent variables of the optimization problem, and (16) as an equality constraint, see $[3,4,20]$ and references therein given. Let $\psi\left(j_{0}\right)$ be the total absolute electric current function given by

$$
\psi\left(j_{0}\right)=\left\|j_{0}\right\|_{L_{1}(\Omega)}=\int_{\Omega}\left|j_{0}\right| d x .
$$

We propose here the following penalized SAND formulation of the inverse EMC problem:

$$
\begin{aligned}
& \min _{j_{0}, \phi, c} J(\phi)+\rho \psi\left(j_{0}\right), \\
& \text { s.t. }\left\{\begin{aligned}
-\Delta \phi & =\mu_{0} j_{0} & & \text { in } \Omega, \\
\frac{\partial \phi}{\partial n} & =\varkappa \bar{p} & & \text { on } \Gamma, \\
\phi(x) & =c+o(1) & & \text { as }\|x\| \rightarrow \infty,
\end{aligned}\right. \\
& \int_{\Omega} j_{0} d x=0, \\
& \left|j_{0}\right| \leq I \text {, }
\end{aligned}
$$

where $I$ is a given bound for the electric current density, and $\rho \psi\left(j_{0}\right)$ acts as a penalty term depending on the penalty parameter $\rho$. Formulation (18) relaxes the considered in [5], where $j_{0}$ was required to be a $0-1$ solution, i.e. to satisfy $j_{0}(x) \in\{-I, 0, I\}$ at each $x \in \Omega$. However, we will show numerically that the addition of the penalty term produces three important beneficial effects on the solution: (i) it produces regularization of the solution, (ii) it penalizes solutions 
with high total absolute electric current, and (iii) it conduces naturally to 0-1 solutions, so avoiding intermediate values of $j_{0}$ in the set $(-I, 0) \cup(0, I)$. We will show also that in the discrete version this penalty term preserves the quadratic programming structure of the original problem. This is very important and unusual in the topology optimization field, where the penalty approaches used to obtain 0-1 solutions, for example when using the SIMP method [21], usually lead to nonconvex formulations with multiple suboptimal local minima [22, 23].

\subsection{The discrete convex quadratic programming formulation}

The solution to the inverse problem must consist of a simple configuration of inductors. In the discrete version of (18) we look for an electric current density distribution $j_{0}$ of the form:

$$
j_{0}=I \sum_{p=1}^{m} \alpha_{p} \chi_{\Theta_{p}}
$$

where $\alpha_{p} \in[-1,1]$ for each $1 \leq p \leq m$, the cells $\Theta_{p}$ are fixed disjoint bounded domains satisfying $\Theta=\cup_{p=1}^{m} \Theta_{p} \subset \Omega$, and $\chi_{\Theta_{p}}$ denotes the characteristic function of $\Theta_{p}$. Note that the electric current density $j_{0}$ is uniform on each $\Theta_{p}$. Inductors made of bundled insulated strands allow the implementation of good approximations to such kind of distribution, see [24] and references therein.

To deal with the exterior boundary value problem of (18), we rely on the following integral equation for the solution $\phi[16,19,25,26]$ :

$$
c(\xi) \phi(\xi)+\int_{\Gamma} q^{*} \phi d s-\int_{\Gamma} u^{*} \varkappa \bar{p} d s=c+\int_{\Omega} u^{*} \mu_{0} j_{0}(x) d x,
$$

where $u^{*}$ is the fundamental solution of the problem, $u^{*}(\xi, x)=-\log \|\xi-x\| /(2 \pi)$, $q^{*}$ is the normal derivative of $u^{*}$, the characteristic function $c(\xi)=1$, for each interior point $\xi$, and $c(\xi)=\Delta \theta /(2 \pi)$, for each point $\xi \in \Gamma$, where $\Delta \theta$ is the angle, internal to $\Omega$, formed by the right and left tangents to $\Gamma$ at $\xi$. The first integral on the left hand side of (20) must be understood in the Cauchy principal value sense.

The spatial discretization consists of approximating the boundary $\Gamma$ into $N$ linear elements $\Gamma_{j}, 1 \leq j \leq N$. The functions $\phi$ and $\varkappa \bar{p}$ are approximated inside each element by piecewise linear polynomials in the form:

$$
\phi(x)=\boldsymbol{N}(x) \phi^{(j)}, \quad \varkappa(x) \bar{p}(x)=\boldsymbol{N}(x) \overline{\boldsymbol{p}}^{(j)}, \quad \text { in } \Gamma_{j},
$$

where $\boldsymbol{N} \in \mathbb{R}^{1 \times 2}$ is the matrix of the linear interpolation functions and $\phi^{(j)}$ and $\overline{\boldsymbol{p}}^{(j)}$ are the vectors that contain the nodal values corresponding to $\phi$ and $\varkappa \bar{p}$ in the element $\Gamma_{j}$. The collocation boundary element method (BEM), builds a linear system imposing (20) at each node $\xi_{i}$ of the boundary:

$$
c_{i} \phi_{i}+\sum_{j=1}^{N} \boldsymbol{h}_{i j} \boldsymbol{\phi}^{(j)}-\sum_{j=1}^{N} \boldsymbol{g}_{i j} \boldsymbol{q}^{(j)}=c+\boldsymbol{A}_{i} \boldsymbol{\alpha}, \quad 1 \leq i \leq N,
$$

where the element matrices $\boldsymbol{h}_{i j}$ and $\boldsymbol{g}_{i j}$ are:

$$
\boldsymbol{h}_{i j}=\int_{\Gamma_{j}} q^{*}(\xi, x) \mathbf{N}(x) d s, \quad \boldsymbol{g}_{i j}=\int_{\Gamma_{j}} u^{*}(\xi, x) \mathbf{N}(x) d s
$$


$\boldsymbol{\alpha} \in \mathbb{R}^{m}$ is the vector with the values $\alpha_{p}$ and the matrix $\boldsymbol{A} \in \mathbb{R}^{N \times m}$ is given by:

$$
\boldsymbol{A}_{i j}=\int_{\Theta_{i}} u^{*}(\xi, x) I \mu_{0} d x
$$

The linear system (22) can be expressed in matrix form as:

$$
\boldsymbol{H} \phi-G \bar{p}=c \boldsymbol{d}+\boldsymbol{A \alpha}
$$

where $\boldsymbol{H} \in \mathbb{R}^{N \times N}$ was assembled from the values of $c_{i}$ and $\boldsymbol{h}_{i j}, \boldsymbol{G} \in \mathbb{R}^{N \times N}$ was assembled from the values of matrices $\boldsymbol{g}_{i j}$, the vectors $\phi \in \mathbb{R}^{N}$ and $\overline{\boldsymbol{p}} \in \mathbb{R}^{N}$ contain all the nodal variables corresponding to $\phi$ and $\varkappa \bar{p}$, respectively, and $\boldsymbol{d} \in \mathbb{R}^{N}$ is the vector with all components equal to one. In addition, we have

$$
\begin{gathered}
J(\phi)=\frac{1}{2} \int_{\Gamma} \phi^{2} d s=\frac{1}{2} \boldsymbol{\phi}^{T} \boldsymbol{M} \boldsymbol{\phi} \\
\int_{\Omega} j_{0} d s=\boldsymbol{e}^{T} \boldsymbol{\alpha}, \quad \psi\left(j_{0}\right)=\int_{\Omega}\left|j_{0}\right| d s=\boldsymbol{e}^{T}|\boldsymbol{\alpha}|,
\end{gathered}
$$

where the sparse matrix $M \in \mathbb{R}^{N \times N}$ is obtained by integrating the interpolation functions and $\boldsymbol{e} \in \mathbb{R}^{m}$ is obtained from (19) and (27).

Finally, by defining the positive and negative parts $\boldsymbol{\alpha}^{+} \in \mathbb{R}^{m}, \boldsymbol{\alpha}^{-} \in \mathbb{R}^{m}$ of $\boldsymbol{\alpha}$ by $\boldsymbol{\alpha}_{p}^{+}=\max \left\{0, \boldsymbol{\alpha}_{p}\right\}$ and $\boldsymbol{\alpha}_{p}^{-}=\max \left\{0,-\boldsymbol{\alpha}_{p}\right\}$, we have $\boldsymbol{\alpha}=\boldsymbol{\alpha}^{+}-\boldsymbol{\alpha}^{-}$, and $|\boldsymbol{\alpha}|=\boldsymbol{\alpha}^{+}+\boldsymbol{\alpha}^{-}$. Therefore, from (25) and (26)-(27) we can formulate a discrete version of (18) as the following convex quadratic programming problem:

$$
\begin{array}{ll}
\min _{\boldsymbol{\alpha}^{+}, \boldsymbol{\alpha}^{-}, \boldsymbol{\phi}, c} & \frac{1}{2} \boldsymbol{\phi}^{T} \boldsymbol{M} \boldsymbol{\phi}+\rho \boldsymbol{e}^{T}\left(\boldsymbol{\alpha}^{+}+\boldsymbol{\alpha}^{-}\right), \\
\text {s.t. } & \boldsymbol{H} \boldsymbol{\phi}-\boldsymbol{G} \overline{\boldsymbol{p}}=c \boldsymbol{d}+\boldsymbol{A}\left(\boldsymbol{\alpha}^{+}-\boldsymbol{\alpha}^{-}\right), \\
& \boldsymbol{e}^{T}\left(\boldsymbol{\alpha}^{+}-\boldsymbol{\alpha}^{-}\right)=0 \\
& 0 \leq \boldsymbol{\alpha}^{+} \leq 1 \\
& 0 \leq \boldsymbol{\alpha}^{-} \leq 1 .
\end{array}
$$

Note that the first $N$ equality constraints are defined by the boundary element matrices $\boldsymbol{H}, \boldsymbol{G}$ and $\boldsymbol{A}$ that are full in the general case. However, if the number of cells is much larger than the number of boundary elements, i.e. if $m>>N$, then Problem (28) is sparse.

\subsubsection{A simple variable mesh approach}

Even thought (28) is a sparse convex quadratic programming problem for which there are very efficient algorithms of solution for large scale problems, the implementation of a variable mesh approach can reduce dramatically the computational costs of solution. As we will show in Section 4, the introduction of the penalty term conduces to regular $0-1$ solutions, with large regions of constant electric current density whose geometric representation can be improved refining the mesh only nearby their boundaries. Hence, the simple variable mesh approach proposed is the following: start with a coarse mesh, solve Problem (28) and refine the mesh subdividing those cells whose dimensionless electric current density $\alpha_{p}$ differs more than a specified tolerance of the corresponding value of any of the adjacent cells, i.e., we subdivide in four smaller cells each cell $\Theta_{p}$ of the mesh having an adjacent cell $\Theta_{q}$ such that $\left|\alpha_{p}-\alpha_{q}\right|>\operatorname{Tol}_{\alpha}$, where $\operatorname{Tol}_{\alpha}$ is the chosen tolerance. In addition, 
for better representing the geometry of $\Theta$, we also subdivide the cells adjacent to its boundary.

\section{Numerical examples}

To show the efficacy of the proposed approach we present results for five examples. In physically compatible units, we have set for all the examples $\sigma=1.0 \times 10^{-4}$ and $\mu_{0}=1.0$. When using the variable mesh approach, the value $\operatorname{Tol}_{\alpha}=1.0 \times 10^{-2}$ was specified. To solve the finite dimensional convex quadratic problems we used the quadprog routine of the Optimization Toolbox of MATLAB. The interiorpoint-convex option with TolFun $=1.0 \times 10^{-18}$ was considered. The numerical experiments were carried out in a laptop PC with an Intel Core i7 M620 $2.67 \mathrm{GHz}$ CPU and $6.0 \mathrm{GiB}$ of RAM.

The target shape of the first example is the same as the considered in the example 2 of [5]. It is the solution of a direct free-surface problem for a liquid metal column of cross-section area $S_{0}=\pi$, with six distributed electric currents of density $I=0.4$ as shown in Fig. 1. Then, the six inductors constitute an optimal solution for the inverse problem. However, from the numerical point of view, this solution is not exactly optimal for the current formulation, since the approaches used to solve the direct and the inverse problems are different. For example, in the approach for the direct problem a weak form of the equilibrium equation was considered, see $[3,4]$. In addition, there is numerical evidence indicating that the solution to the inverse problem is not unique [5]. For these reasons, the optimized solutions are very different from the configuration given in Fig. 1. Figure 2(a) shows the solution obtained for a penalty parameter $\rho=0$ on a fixed mesh of cells of size 0.02. This solution has three main characteristics that are highly undesired for manufacturing purposes: first, it shows an irregular pattern with the electric current density varying from -1 to 1 in the region near the liquid metal. Second, it is not a $0-1$ solution, since outside the region near the liquid metal, the values of $\alpha_{p}$ vary continuously in the range $[-1,1]$. Third, the total absolute electric current $\psi\left(j_{0}\right)$ is high. Figure 2(b) shows the solution obtained for a penalty parameter $\rho=1 \times 10^{-7}$. It is a regular $0-1$ solution for the inverse EMC problem, i.e., regions where $\alpha_{p}$ is one of $\{-1,0,1\}$ are clearly identifiable, and the union of these regions is the entire set $\Theta$. In addition, this solution highly reduces the total absolute electric current. The price to pay is a small increment in the Kohn-Vogelius functional from the value $1.34 \times 10^{-11}$ to $3.50 \times 10^{-10}$ that produces an imperceptible effect on the equilibrium shape as shown in Fig. 2(b). Figure 3 shows the effect of locating the inductors separated from the liquid metal at an increasing distance. While in Fig. 2(b) the inductors are located to a distance $d=0.15$ from the liquid metal, in Fig. 3(a) $d=0.25$ and in Fig. 3(b) $d=0.35$, which is almost the distance of the know solution of Fig. 1(b). As the distance tends to the value $d=0.35$, the solutions look more like the known solution, with the total absolute electric current $\psi\left(j_{0}\right)=0.222$ in the case $d=0.15, \psi\left(j_{0}\right)=0.274$ for $d=0.25$ and $\psi\left(j_{0}\right)=0.349$ for $d=0.35$, which is still less that the value $\psi\left(j_{0}\right)=0.384$ of the known solution. The solutions found are then more economical than the known solution, and this fact is due to the addition of the penalty term. Figure 4 shows the importance of choosing an appropriate penalty functional. Figure 4(a) presents the solution obtained by penalizing $\psi\left(j_{0}\right)=\left\|j_{0}\right\|_{L_{1}(\Omega)}$, while Fig. $4(\mathrm{~b})$ shows the solution obtained by penalizing $\left\|j_{0}\right\|_{L_{2}(\Omega)}^{2}=\int_{\Omega}\left|j_{0}\right|^{2} d x$. The penalty parameters were chosen to obtain similar values for the Kohn-Vogelius functional. Note that the second functional leads to a regular but not a $0-1$ solution. Indeed, the values 


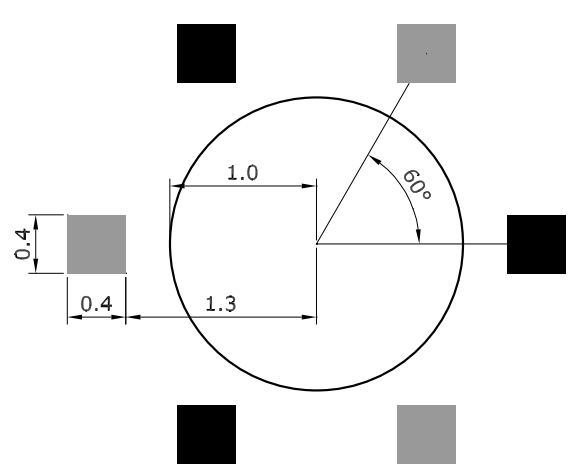

(a)

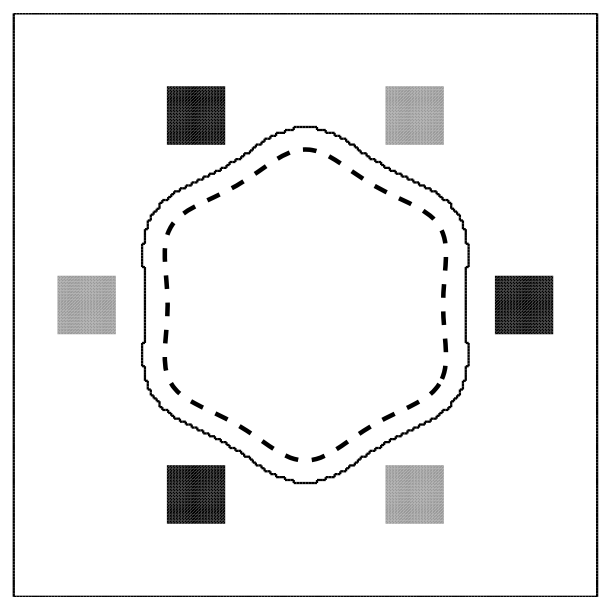

(b)

Figure 1. Example 1- (a) initial configuration of the direct free-surface problem, (b) target shape of area $S_{0}=\pi$. Black area: positive inductors, gray area: negative inductors, dashed line: target shape, thin solid line: boundary of the mesh of cells.

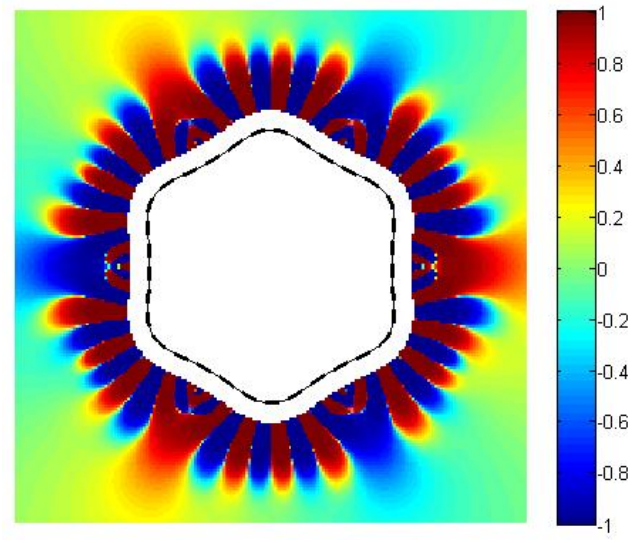

(a)

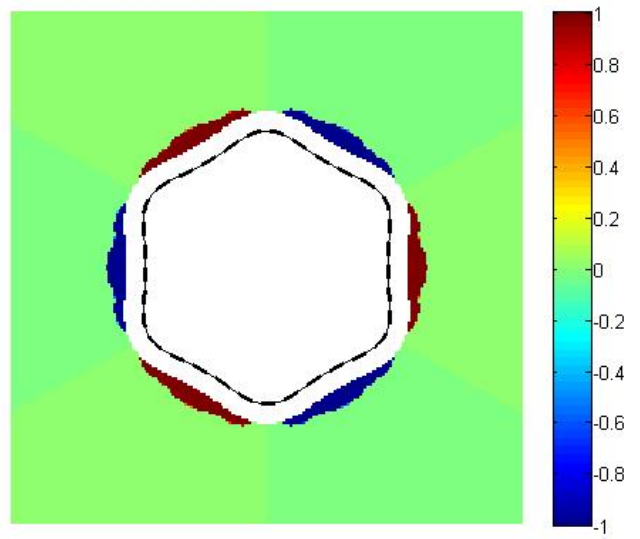

(b)

Figure 2. Example 1 - contour plot of $I^{-1} j_{0}$, (a) $\rho=0$, (b) $\rho=1 \times 10^{-7}$. Dashed line: target shape, thin solid line: equilibrium shape obtained for the optimized inductors.

of the electric current density does not reach the bounds.

In the second example we have moved the inductors of the previous example to generate the asymmetric target shape shown in Fig. 5. This problem was solved for $I=0.4$ and $\rho=1 \times 10^{-7}$ using both a fixed mesh of cells of size 0.0125 and the variable mesh approach, starting from a mesh of cells of size 0.4 and performing subsequent mesh refinements until the smallest cells are of size 0.0125 . The solutions obtained by both approaches are almost the same, with the difference that the first approach solved a problem of 75433 cells, taking 934 seconds to obtain the solution, while the second approach solved a problem with only 5728 cells in the final mesh, taking only 93 second to solve all the problems from the initial to the final mesh. Figure 6 shows the solution obtained using the variable mesh approach.

The third example corresponds to the equilibrium shape of the interior problem shown in Fig. 7. In this case $S_{0}=1.0, I=50.0$ and $\rho=1 \times 10^{-7}$. The problem was solved using the variable mesh approach and the solution is shown in Fig. 8. This example demonstrates the ability of the current approach to find an economical solution. The total absolute electric current is $\psi\left(j_{0}\right)=2.784$ for the solution obtained and $\psi\left(j_{0}\right)=4.0$ for the known solution of Fig. 7 (a). However, note that, 
10

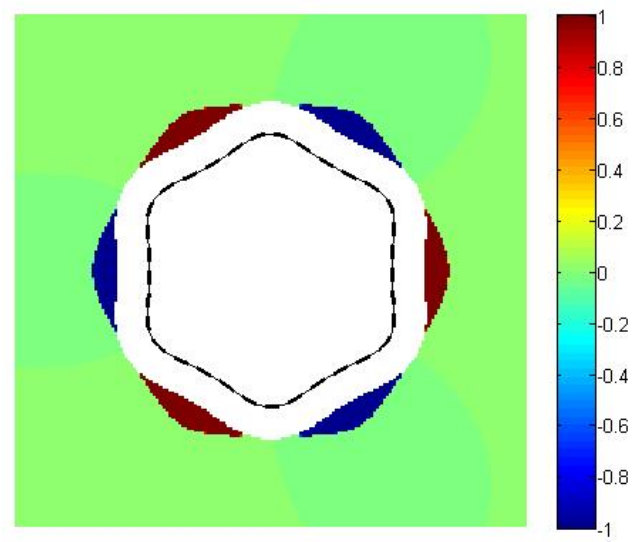

(a)

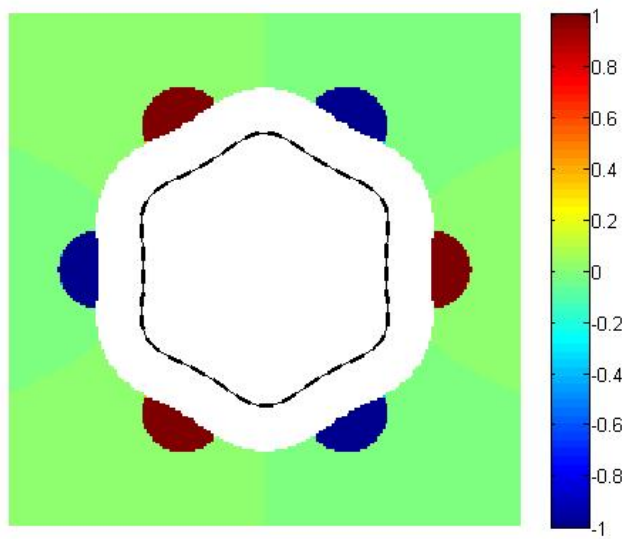

(b)

Figure 3. Example 1 - contour plot of $I^{-1} j_{0}$, (a) inductors located to a distance $d=0.25$ from the liquid metal, (b) $d=0.35$. Dashed line: target shape, thin solid line: equilibrium shape obtained for the optimized inductors.

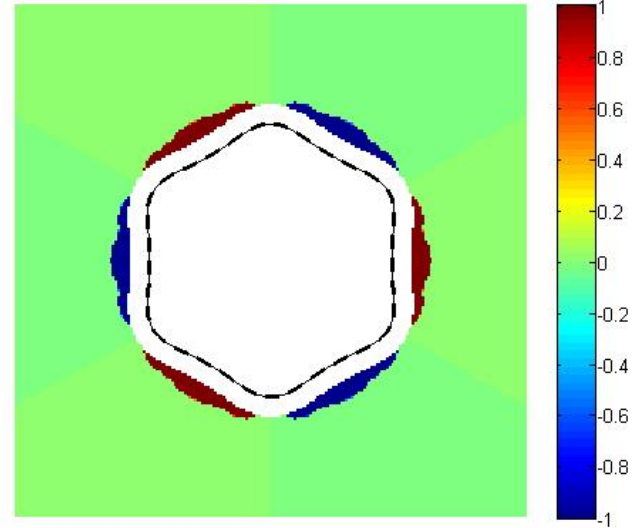

(a)

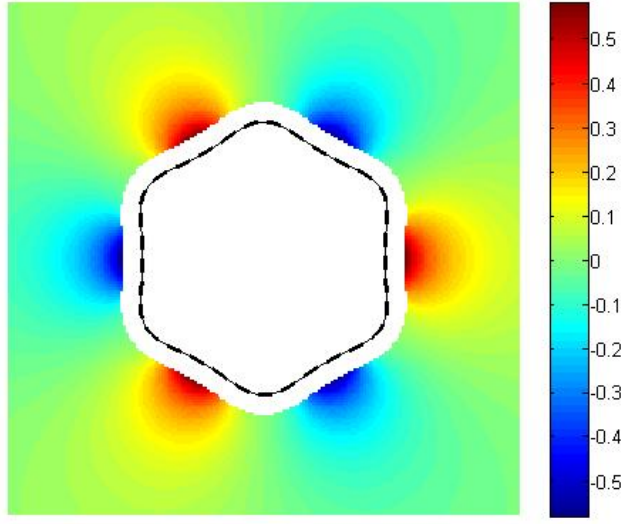

(b)

Figure 4. Example 1 - contour plot of $I^{-1} j_{0}$, (a) penalizing $\left\|j_{0}\right\|_{L_{1}(\Omega)}$, (b) penalizing $\left\|j_{0}\right\|_{L_{2}(\Omega)}^{2}$. Dashed line: target shape, thin solid line: equilibrium shape obtained for the optimized inductors.

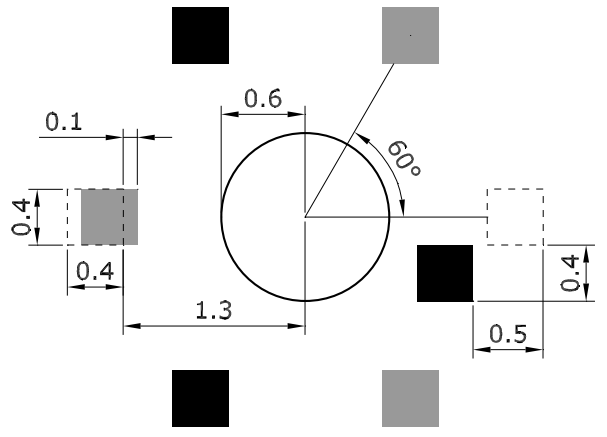

(a)

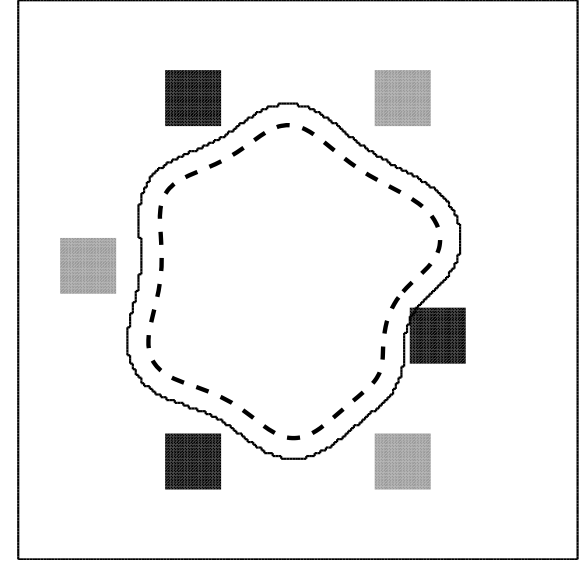

(b)

Figure 5. Example 2 - (a) initial configuration of the direct free-surface problem, (b) target shape of area $S_{0}=\pi$. Black area: positive inductors, gray area: negative inductors, dashed line: target shape, thin solid line: boundary of the mesh of cells. 


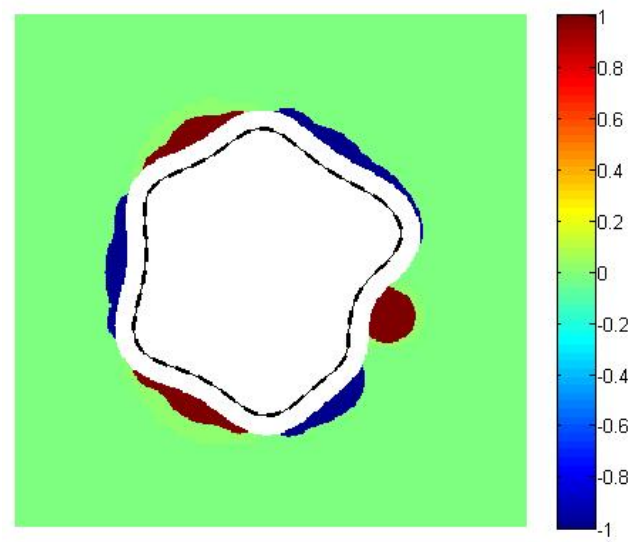

(a)

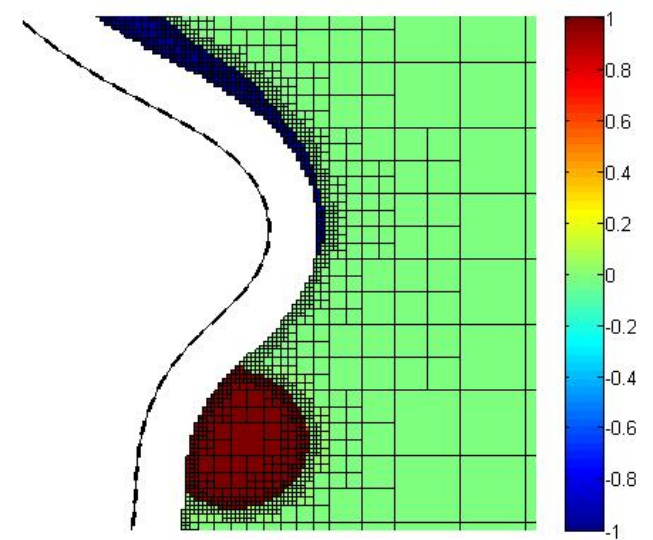

(b)

Figure 6. Example 2 - contour plot of $I^{-1} j_{0}$. Solution obtained using a variable mesh. Dashed line: target shape, thin solid line: equilibrium shape obtained for the optimized inductors.

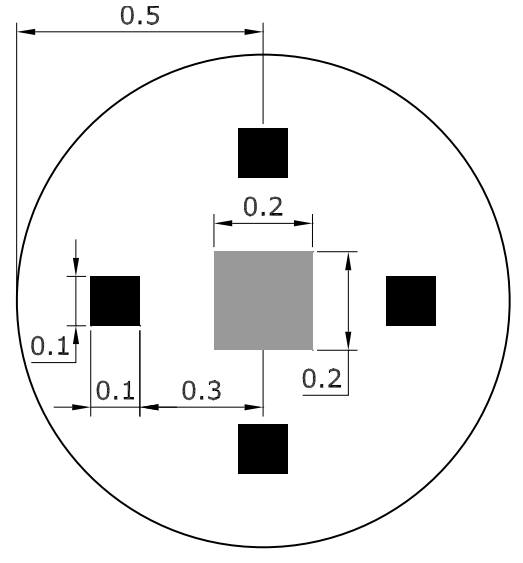

(a)

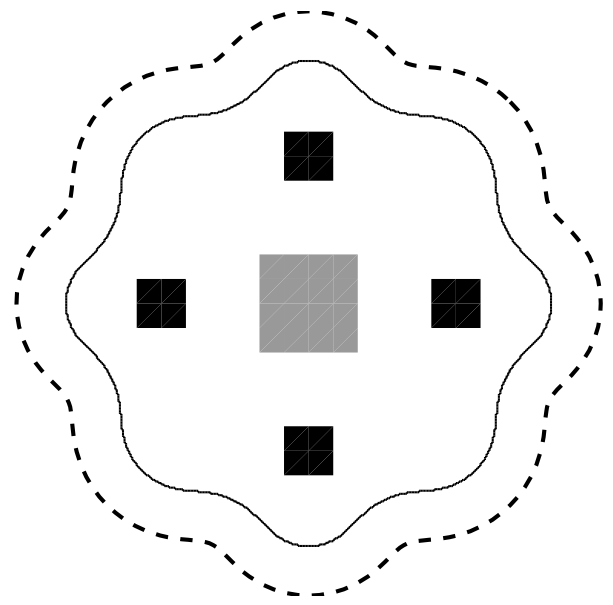

(b)

Figure 7. Example 3 - (a) initial configuration of the direct free-surface problem, (b) target shape of area $S_{0}=1$. Black area: positive inductors, gray area: negative inductors, dashed line: target shape, thin solid line: boundary of the mesh of cells.

unlike the previous exterior problems, the solution of this interior problem presents a spatial pattern of electric current density of alternating sign. We have seen that the alternating pattern cannot be removed by increasing the penalty parameter $\rho$, by refining the boundary mesh of the liquid metal surface, or by refining the mesh of cells. It also remains if we consider $\Theta$ as a circle as shown in Fig. 9 were we can see that the distance to the liquid metal strongly influences the value of the total absolute electric current which is $\psi\left(j_{0}\right)=1.875$ in Fig. $9(\mathrm{a})$, and $\psi\left(j_{0}\right)=6.103$ in Fig. 9(b). Further studies should clarify the alternating sign pattern phenomenon, that we have not observed in the solution of exterior problems when using the penalty approach.

The fourth and the fifth examples are truly inverse problems defined by target shapes that are not obtained as solutions of direct problems. The fourth example is an exterior problem defined by the target shape depicted in Fig. 10, that was also studied in [5]. Here we solved this problem for $I=0.2$ and $\rho=1 \times 10^{-7}$, using the variable mesh approach. Figure 11 shows the solution obtained.

The final example corresponds to the interior problem with the target shape of Fig. 12. For $I=4.0, \rho=1 \times 10^{-7}$ and using the variable mesh approach, the 


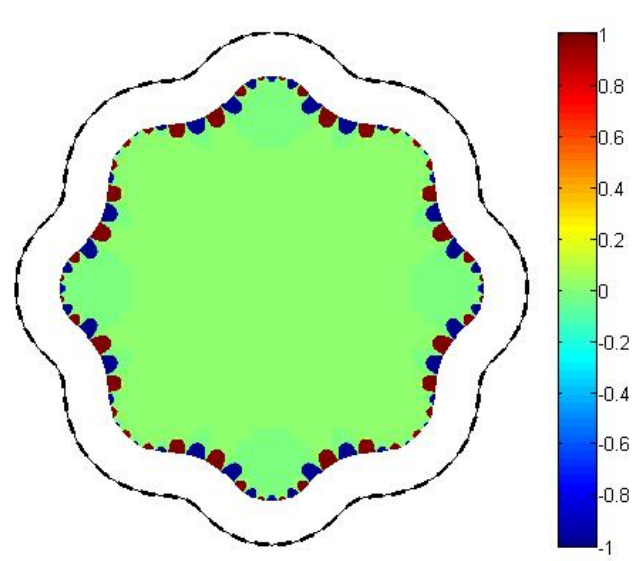

(a)

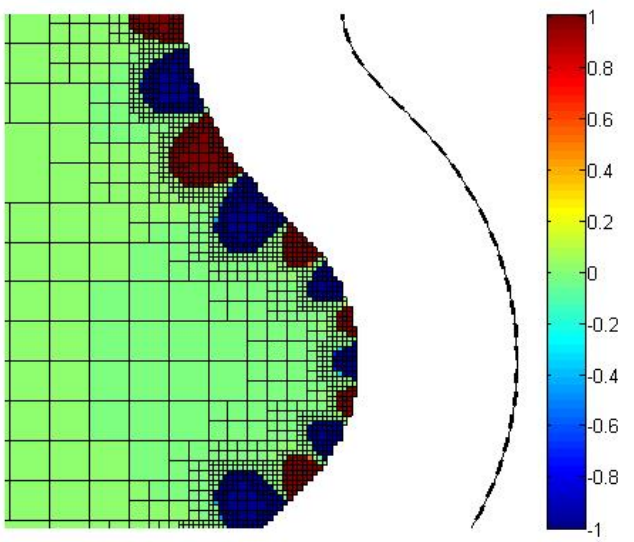

(b)

Figure 8. Example 3 - contour plot of $I^{-1} j_{0}$. Solution obtained using a variable mesh. Dashed line: target shape, thin solid line: equilibrium shape obtained for the optimized inductors.

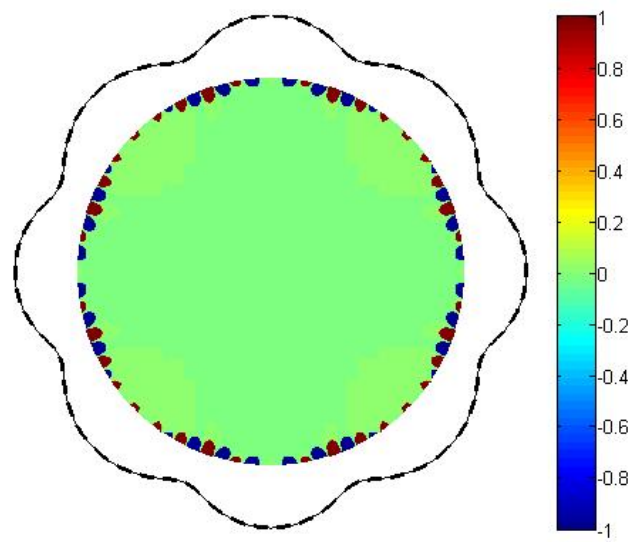

(a)

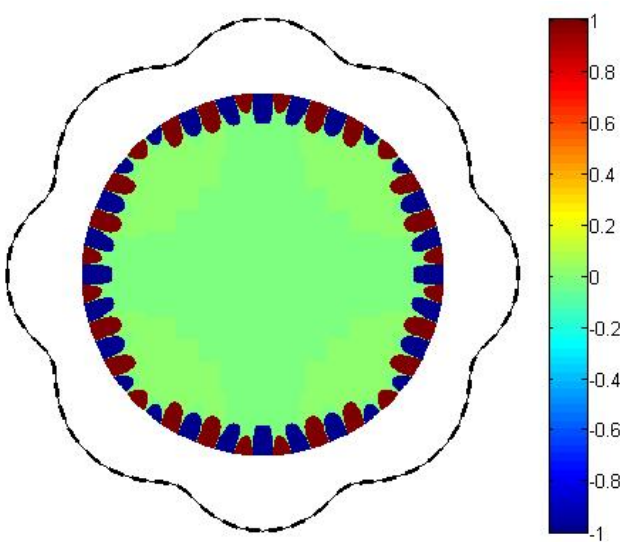

(b)

Figure 9. Example 3 - contour plot of $I^{-1} j_{0}$. Result for a circular $\Theta$ of radius a) $r=4.5$, b) $r=4.2$. Solution obtained using a variable mesh. Dashed line: target shape, thin solid line: equilibrium shape obtained for the optimized inductors.

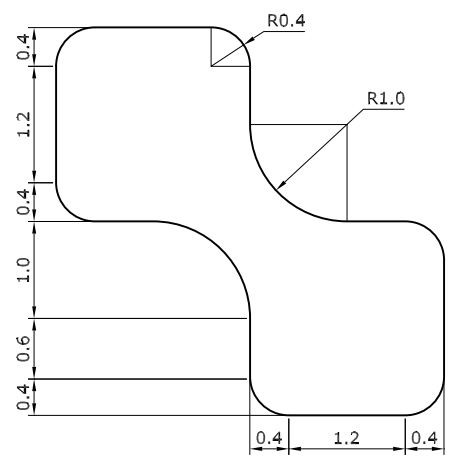

(a)

(b)

Figure 10. Example 4 - (a) description of the problem geometry, (b) target shape. Dashed line: target shape, thin solid line: boundary of the mesh of cells. 


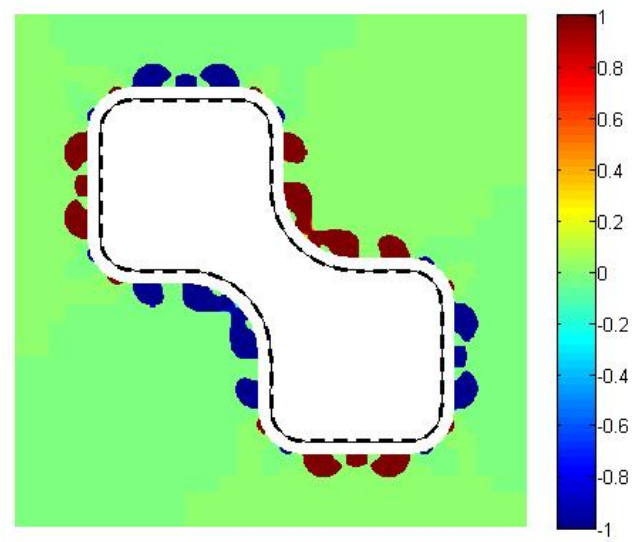

(a)

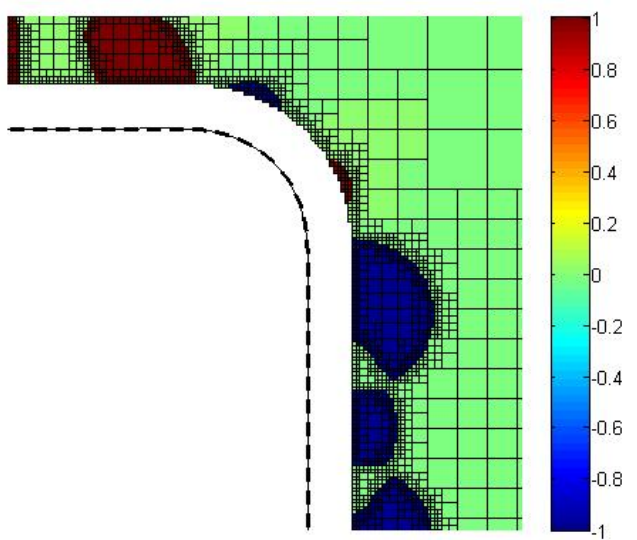

(b)

Figure 11. Example 4 - contour plot of $I^{-1} j_{0}$. Solution obtained using a variable mesh. Dashed line: target shape, thin solid line: equilibrium shape obtained for the optimized inductors.

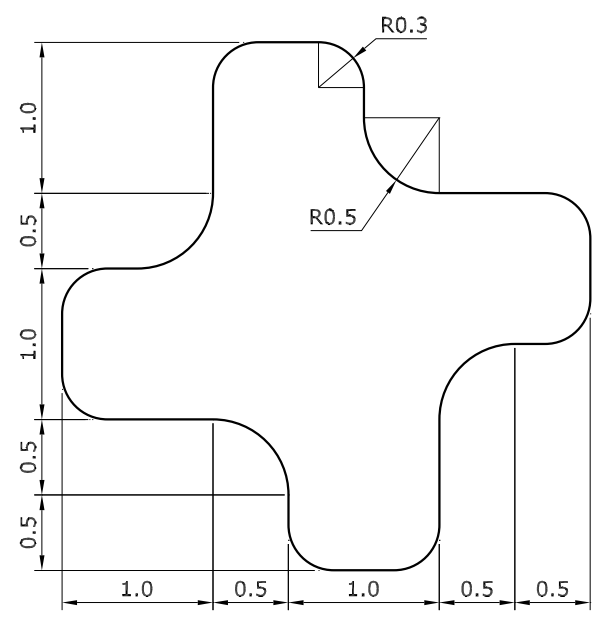

(a)

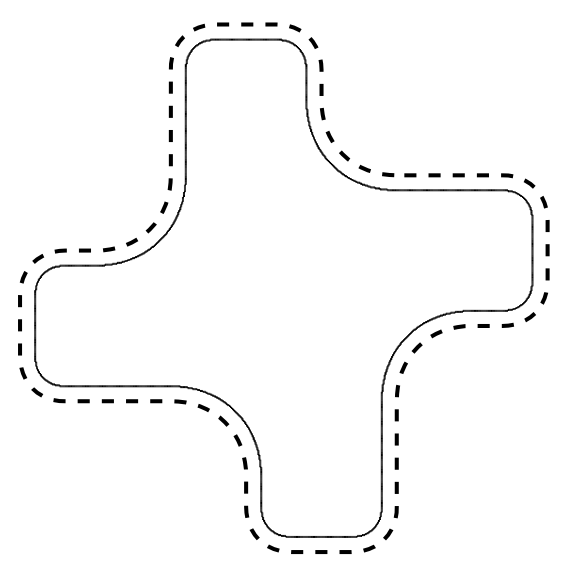

(b)

Figure 12. Example 5 - (a) description of the problem geometry, (b) target shape. Dashed line: target shape, thin solid line: boundary of the mesh of cells.

solution is shown in Fig. 13. Note the pattern of alternating electric current sign observed also in the solutions of the interior problems of Figs. 8 and 9.

\section{Conclusions}

A new topology optimization method for solving an inverse problem concerning EMC problems have been presented. We have taken a recently proposed topology optimization formulation based on the Kohn-Vogelius criterion, and reformulated it using the SAND approach as an sparse convex quadratic programming problem. The sparse convex quadratic programming nature of the discrete problems allows the use of very efficient interior-point algorithms, and a simple variable mesh approach have been proposed to obtain an additional reduction of the computational costs of solution.

The results presented for some numerical examples show that the addition of an appropriate penalty term, that preserves the quadratic programming structure of the problem, conduces to regular $0-1$ solutions with low absolute total electric 


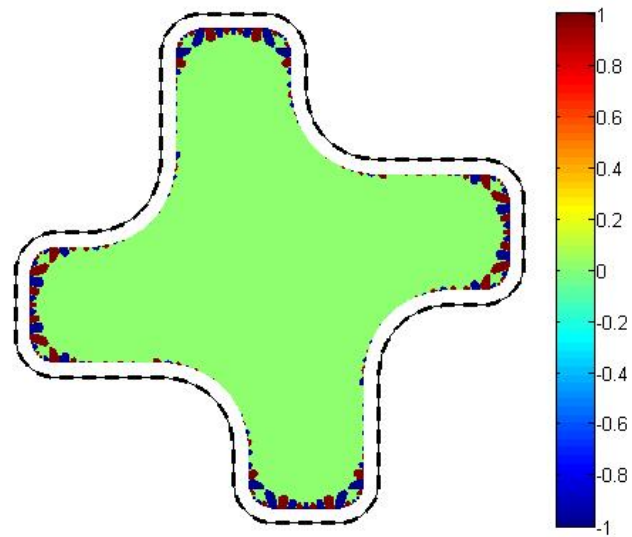

(a)

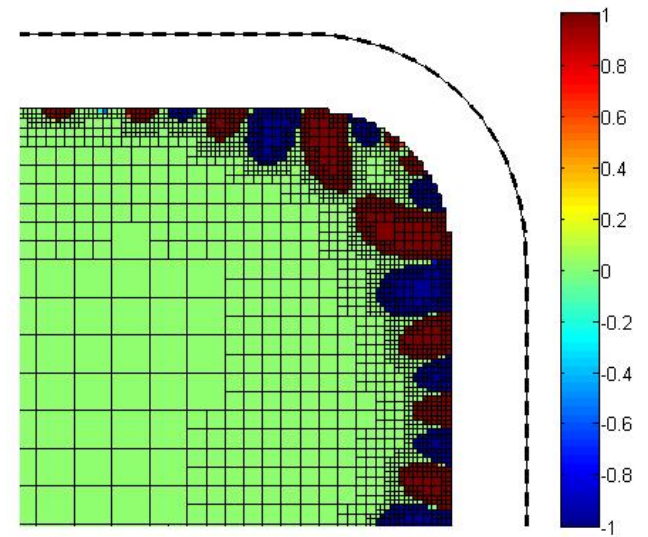

(b)

Figure 13. Example 5 - contour plot of $I^{-1} j_{0}$. Solution obtained using a variable mesh. Dashed line: target shape, thin solid line: equilibrium shape obtained for the optimized inductors.

current. This characteristics set the present approach as optimal for the considered model of the inverse EMC problem.

\section{Acknowledgments}

The authors thank the Brazilian Research Councils CAPES, CNPq, FAPERJ, the institutions supporting the program Ciência Sem Fronteiras of Brazil, the Uruguayan Research Councils ANII and CSIC and the French Research Councils CNRS and INRIA for the financial support.

\section{References}

[1] J.P. Brancher and O.E. Séro-Guillaume, Étude de la déformation d'un liquide magnétique, Archive for Rational Mechanics and Analysis 90 (1985), pp. 57-85.

[2] A. Gagnoud, J. Etay, and M. Garnier, Le problème de frontière libre en lévitation électromagnétique, Journal de Mécanique Théorique et Appliquée 5 (1986), pp. 911-934.

[3] A. Canelas, J.R. Roche, and J. Herskovits, The inverse electromagnetic shaping problem, Structural and Multidisciplinary Optimization 38 (2009), pp. 389-403.

[4] - Inductor shape optimization for electromagnetic casting, Structural and Multidisciplinary Optimization 39 (2009), pp. 589-606.

[5] A. Canelas, A.A. Novotny, and J.R. Roche, A new method for inverse electromagnetic casting problems based on the topological derivative, Journal of Computational Physics 230 (2011), pp. 3570-3588.

[6] J.D. Jackson Classical electrodynamics, Third Wiley, 1998.

[7] A. Henrot and M. Pierre, Un problème inverse en formage des métaux liquides, RAIRO Modélisation Mathématique et Analyse Numérique 23 (1989), pp. 155-177.

[8] H.K. Moffatt, Magnetostatic equilibria and analogous Euler flows of arbitrarily complex topology. Part 1. Fundamentals, Journal of Fluid Mechanics 159 (1985), pp. 359-378.

[9] A. Novruzi and J.R. Roche, Second Order Derivatives, Newton Method, Application to Shape Optimization, RR-2555, INRIA, 1995.

[10] M. Pierre and J.R. Roche, Computation of free surfaces in the electromagnetic shaping of liquid metals by optimization algorithms, European Journal of Mechanics. B Fluids 10 (1991), pp. 489-500.

[11] J.A. Shercliff, Magnetic Shaping of Molten Metal Columns, Proceedings of the Royal Society of London. Series A, Mathematical and Physical Sciences 375 (1981), pp. 455-473.

[12] M. Pierre and J.R. Roche, Numerical simulation of tridimensional electromagnetic shaping of liquid metals, Numerische Mathematik 65 (1993), pp. 203-217.

[13] J.R. Roche, Gradient of the discretized energy method and discretized continuous gradient in electromagnetic shaping simulation, Applied Mathematics and Computer Science 7 (1997), pp. 545-565.

[14] $363-377$.

[15] A. Novruzi and J.R. Roche, Newton's method in shape optimisation: A three-dimensional case, BIT. Numerical Mathematics 40 (2000), pp. 102-120.

[16] K.E. Atkinson The numerical solution of integral equations of the second kind, Cambridge Monographs on Applied and Computational Mathematics Vol. 4, Cambridge University Press, Cambridge, 1997. 
[17] J.C. Nédélec Acoustic and Electromagnetic Equations. Integral Representations for Harmonic Problems, Applied Mathematical Sciences Vol. 144, Springer-Verlag, New York, 2001.

[18] T.P. Felici and J.P. Brancher, The inverse shaping problem, European Journal of Mechanics. B Fluids 10 (1991), pp. 501-512.

[19] G. Hsiao and W.L. Wendland Boundary Integral Equations, Applied Mathematical Sciences Vol. 164, Springer-Verlag, Berlin, 2008.

[20] A. Canelas, J. Herskovits, and J.C.F. Telles, Shape optimization using the boundary element method and a SAND interior point algorithm for constrained optimization, Computers \& Structures 86 (2008), pp. 1517-1526 Structural Optimization.

[21] G.I.N. Rozvany, Aims, scope, methods, history and unified terminology of computer-aided topology optimization in structural mechanics, Structural and Multidisciplinary Optimization 21 (2001), pp. 90-108.

[22] M.P. Bendsøe Optimization of structural topology, shape, and material, Springer-Verlag, Berlin, 1995.

[23] H.A. Eschenauer and N. Olhoff, Topology optimization of continuum structures: A review, Applied Mechanics Reviews 54 (2001), pp. 331-390.

[24] C.R. Sullivan, Optimal Choice for Number of Strands in a Litz-Wire Transformer Winding, IEEE Transactions On Power Electronics 14 (1999), pp. 283-291.

[25] C.A. Brebbia, J.C.F. Telles, and L.C. Wrobel Boundary Element Technique: Theory and Applications in Engineering, Springer-Verlag, 1984.

[26] C.A. Brebbia and J. Dominguez Boundary elements: an introductory course, Second Computational Mechanics Publications, Southampton, 1992. 